\title{
Conductivity Characterization of Insulation and Its Effects on the Calculation of the Electric Field Distribution in HVDC Cables
}

\author{
Shili Liu, ${ }^{1}$ Wei Wei, ${ }^{1}$ Tao Liu, ${ }^{1}$ Zhaoyu Hui, ${ }^{1}$ Yuhua Hang, ${ }^{1}$ Huan Zheng $\mathbb{D},{ }^{2}$ \\ Changyou Suo $\left(\mathbb{D},{ }^{2}\right.$ and Zhonghua $\mathrm{Li}\left(\mathbb{C}^{2}\right.$ \\ ${ }^{1}$ Suzhou Nuclear Power Research Institute, Suzhou 215004, China \\ ${ }^{2}$ Key Laboratory of Engineering Dielectrics and Its Application, Ministry of Education, \\ Harbin University of Science and Technology, Harbin 150080, China \\ Correspondence should be addressed to Changyou Suo; cysuo@hrbust.edu.cn
}

Received 22 October 2020; Revised 14 January 2021; Accepted 29 January 2021; Published 17 February 2021

Academic Editor: Bekir Sahin

Copyright ( $\odot 2021$ Shili Liu et al. This is an open access article distributed under the Creative Commons Attribution License, which permits unrestricted use, distribution, and reproduction in any medium, provided the original work is properly cited.

\begin{abstract}
The calculation of an electric field distribution provides the basis for the structural design of the insulation, and an accurate characterization of conductivity as a function of temperature and electric field forms an important basis for the simulation of the electric field distribution in HVDC (high-voltage direct current) cables. However, the conductivity functions that describe the insulating materials used for HVDC cables in different studies are different, and very little has been reported regarding how to choose the most accurate function. In this work, the conductivity of insulating materials used for HVDC cables is characterized, and the effects of the conductivity characterization on the simulation of the electric field in HVDC cables are studied. First, eight common conductivity functions are compared qualitatively. Then, the conductivities of XLPE for different temperatures and electric fields are measured, and a data fitting technique is used to analyze the coincidence degree between different functions and the test results. Finally, the steady-state electric field distributions of HVDC cables for different temperature gradients are simulated in COMSOL Multiphysics. The results show that the sum of the square of the relative errors of the fitting when using the original functions is larger than that achieved when using the logarithmic form of the functions. The deviations in the electric field caused by taking the logarithm of different functions are smaller.
\end{abstract}

\section{Introduction}

Extruded high-voltage direct current (HVDC) cables have received wide attention since being introduced because of many advantages such as a simpler production process, lower loss, longer transmission distance, and the constitution of an asynchronous interconnection [1-4]. As a result, many development projects have been launched around the whole world. The number and voltage level of HVDC cables have been increasing continuously in the past 20 years to meet the transmission capacity demand [5-9]. In research aiming to improve the voltage level, most scholars have studied material modifications and cable structural design. The electric field in the insulation is also a main reference standard and needs to be calculated for structural design $[10,11]$. It is well known that the electric field distribution in the insulation of HVDC cables is determined by the conductivity of the insulating material, which is known to be dependent on the electric field and temperature [12-14]. Therefore, obtaining accurate testing data and a characterization function for conductivity is essential for precisely calculating the electric field in the insulation of HVDC cables.

As for the relationship between the conductivity, temperature, and electric field strength of insulating materials, some specific conductivity functions have been given in the literature, but the functions in different studies are not identical [15-20]. Boggs [15] used the characteristic function of a hopping conductance mechanism to calculate the electric field in the insulation of HVDC cables and studied the influence of different dependent coefficients of the electric field and activation energy on the electric field 
distribution. Mazzanti et al. [16] used two empirical conductivity functions to calculate the electric field in insulation and studied the relation between the coefficients of the electric field dependence of the two functions. Liu et al. [17] calculated the electric field distribution in XLPE insulation of HVDC cables under time-varying states with a hopping conduction model and analyzed the relation between the electric field distribution and the insulation conductivity. Gao et al. [18] measured the conductivity of an XLPE nanocomposite and polymer-filled XLPE and calculated the thermal activation energies according to a hopping conduction model. The authors found that the addition of a nanofiller changes the activation energy less than a polymer filler. Up to now, scholars have not reached a consensus regarding the conductivity functions. Even when acquiring an exact conductivity testing value of a material, the electric field will be still different if it is calculated by using different conductivity functions. The difference in the functions and finding the most accurate characteristic function are worth studying.

The purpose of the work presented in this paper is to explore the difference in the conductivity characteristics of insulation and its effects on the simulation of an electric field distribution because the simulation of an electric field distribution provides the basis for the design of an insulation structure. Cross-linked polyethylene (XLPE), as one of the common materials used for HVDC cable insulation, is studied in this paper. The DC conductivities are measured, and then the data are fitted by Origin software using different conductivity functions. Finally, a model of an HVDC cable is constructed in COMSOL Multiphysics, and the electric field distribution in the cable insulation is calculated according to the conductivity functions obtained by the fitting.

\section{Theoretical Analysis of Conductivity Characterization Functions}

2.1. Conductivity Characterization. According to classic dielectric physics textbook [21], the conductance mechanisms of solid dielectrics are described by the Poole-Frenkel model, Schottky model, and hopping conductance model, and the corresponding conductivity expressions are, respectively [21-23]:

$$
\begin{aligned}
\gamma_{p f}(E, T) & =\gamma_{p f, 0} \exp \left(-\frac{\varphi \cdot q}{2 k_{b} T}\right) \exp \left(\frac{q \sqrt{E}}{k_{b} T} \sqrt{\frac{q}{4 \pi \varepsilon_{0} \varepsilon_{r}}}\right), \\
\gamma_{s}(E, T) & =\frac{\gamma_{s, 0} T^{2} \exp \left(-\left(\varphi \cdot q / 2 k_{b} T\right)\right) \exp \left(\left(q \sqrt{E} / k_{b} T\right) \sqrt{\left(q / 4 \pi \varepsilon_{0} \varepsilon_{r}\right)}\right)}{E},
\end{aligned}
$$

$\gamma_{h}(E, T)=\frac{\gamma_{h, 0} \exp \left(-\left(\varphi \cdot q / k_{b} T\right)\right) \sinh \left(q d E / 2 k_{b} T\right)}{E}$,

where $T$ is the temperature in Kelvin, $E$ is the electric field in $\mathrm{V} / \mathrm{m}, \gamma_{i}$ are the conductivities for a certain electric field and temperature in $\mathrm{S} / \mathrm{m}, \varphi$ is the thermal activation energy of the conductivity in $\mathrm{eV}, q$ is the electron charge $\left(1.602 \times 10^{-19{ }^{\circ}} \mathrm{C}\right)$, $k_{b}$ is Boltzmann's constant $\left(1.38 \times 10^{-23} \mathrm{~J} / \mathrm{K}\right), \quad \varepsilon_{0}$ is the permittivity of vacuum $\left(0.85 \times 10^{-12} \mathrm{~F} / \mathrm{m}\right), \varepsilon_{r}$ is the relative permittivity, and $d$ is the jumping distance.

The above three characterization functions ((1)-(3)) present specific physical mechanisms. According to the literature, the following five simplified empirical functions ((4)-(8)) are usually used to characterize the conductivity of an insulating material in engineering applications. In addition, there might be other characterization formulas that are not listed here [15, 23-29].

$$
\begin{aligned}
& \gamma_{1}(E, t)=\frac{\gamma_{1,0} \exp \left(-\left(\varphi \cdot q / k_{b} T\right)\right) \sinh (B E)}{E}, \\
& \gamma_{2}(E, t)=\gamma_{2,0} \exp \left(-\frac{a}{T}+b E\right), \\
& \gamma_{3}(E, t)=\gamma_{3,0} \exp (\alpha t+b E), \\
& \gamma_{4}(E, T)=\gamma_{4,0} \exp \left(-\frac{\varphi \cdot q}{k_{b} T}\right) E^{\beta}, \\
& \gamma_{5}(E, t)=\gamma_{5,0} \exp (\alpha t) E^{\beta},
\end{aligned}
$$

where $t$ is the temperature in centigrade, $a$ and $\alpha$ are the coefficients of the temperature dependence, and $B, b$, and $\beta$ are the coefficients of the electric field dependence. The meanings of the other physical quantities are the same as those of the quantities in formulas (1)-(3).

From formulas (4)-(8), it can be seen that the electric field functions are exponential, hyperbolic sine, and power, and the temperature functions are only exponential, only differing in Kelvin or degree centigrade. These functions are simple and indicate that the conductivity increases nonlinearly with the electric field and the temperature. The common characteristics of these functions can be expressed as a product of a temperature function and an electric field function, that is:

$$
\gamma_{i}(E, T)=\gamma_{i, 0} f_{i}(T) g_{i}(E) .
$$

For the above formulas:

$$
\begin{aligned}
f_{1}(T) & =f_{4}(T)=\exp \left(-\frac{\varphi \cdot q}{k_{b} T}\right), \\
f_{2}(T) & =\exp \left(-\frac{a}{T}\right) \\
f_{3}(t) & =f_{5}(t)=\exp (\alpha t), \\
g_{1}(E) & =\frac{\sinh (B E)}{E} \\
g_{2}(E) & =g_{3}(E) \\
& =\exp (b E) \\
g_{4}(E) & =g_{5}(E) \\
& =E^{\beta} .
\end{aligned}
$$


The conductivity can be expressed as a product of a temperature function and an electric field function, which also implies that the temperature and the electric field have no synergistic effects on the conductivity.

2.2. Analysis of the Relationship between Empirical and Theoretical Formulas. The above five empirical formulas $((4)-(8))$ are obtained by a simplification in the derivation of the theoretical formulas, and there are some errors between the empirical and theoretical formulas. The detailed analysis of the errors is shown in the following.

Empirical function (4) is more similar to function (3) in terms of the functional form. The temperature function of function (4) is the same as that of function (3), just $q d /(2 k b T)$ is replaced by the variable B in the electric field function. However, compared with functions (1) and (2), the electric field functions changes from exponential to hyperbolic sine. Thus, it can be inferred that the error between function (4) and function (3) is lower than the error between function (4) and function (1) or (2).

For empirical function (5), its temperature function is similar to that of functions (1), (2), and (3). The electric field function of empirical function (5) is exponential, similar to functions (1) and (2) but not function (3). Therefore, the error between function (5) and function (3) is greater than the error between function (5) and function (1) or (2), which is different from empirical function (4).

The electric field functions of empirical function (6) and function (5) are the same, and their temperature functions are different in the form of the temperature in Kelvin or centigrade degree. If the temperature in function (5) is $t$ in centigrade degree, the temperature function can be written as follows:

$$
f(t)=\exp \left(-\frac{a}{273+t}\right)=\exp \left[\frac{a(273-t)}{273^{3}-t^{2}}\right] .
$$

When $t^{2}$ is ignored,

$$
f(t) \approx \exp \left(-\frac{a}{273}+\frac{a t}{273^{2}}\right)=k \exp (\alpha t),
$$

where $k=\exp (-(a / 273))$ and $\alpha=a / 273^{2}$.

Formula (12) is the temperature function of formula (6). In the derivation process, it can be seen that formula (6) can be regarded as a transformation of the basis of formula (5), and the temperature function is approximately simplified. Therefore, the error of the formula (6) is larger than that of formula (5).

The temperature functions of empirical functions (7) and (8) have been analyzed, and their electric field functions are power functions. Compared to a hyperbolic sine or exponential function, a power function has no physical meaning, leading to larger errors. In addition, the temperature function of formula (8) is simplified on the basis of the formula (7). Therefore, the error of formula (8) is larger than that of formula (7).

\section{Analysis of Conductivity Experiment Results}

3.1. Specimen Preparation and Conductivity Testing. Type LD200 LDPE, which is produced by the China Petroleum and Chemical Corporation, is taken as the basic material. Type LD200 LDPE is mixed with a certain proportion of dicumyl peroxide (DCP), which is a crosslinking reagent produced by Sinopec Shanghai Gaoqiao Petrochemical Company, in a Bunbury mixer at $383 \mathrm{~K}$ for $30 \mathrm{~min}$ to make an XLPE blank. Then, a $2.2 \mathrm{~g}$ blank is placed into a plate vulcanization machine to hot press plate samples of XLPE with a size of $10 \times 10 \mathrm{~mm}$ and a thickness of $0.2 \mathrm{~mm}$. After that, the samples are placed in a plate vulcanizing machine at $448 \mathrm{~K}$ for $30 \mathrm{~min}$ to crosslink the polymer, and the samples are removed after naturally cooling down to room temperature. According to the requirement of a threeelectrode testing system, aluminum electrodes are evaporated on both sides of the XLPE specimens. Finally, the specimens are short-circuited in a vacuum oven at $353 \mathrm{~K}$ for 24 hours to remove some byproducts and charges in the specimens.

The conductivity test system includes a DC high-voltage power supply (HB-Z103-2AC; its output voltage is continuously adjustable from 0 to $10 \mathrm{kV})$, a three-electrode system, an EST122 electrometer with a testing range from $10^{-2}$ to $10^{-14} \mathrm{~A}$, and an oven with a highest working temperature of $473 \mathrm{~K}$ (temperature fluctuation range of $\pm 1 \mathrm{~K}$ ). The conductance currents are measured under nine field strengths ranging from $10 \mathrm{kV} / \mathrm{mm}$ to $50 \mathrm{kV} / \mathrm{mm}$, at $295 \mathrm{~K}$, $313 \mathrm{~K}, 328 \mathrm{~K}, 343 \mathrm{~K}, 358 \mathrm{~K}$, and $368 \mathrm{~K}$. To ensure the accuracy of the conductivity data, the conductivity results are averaged by testing four different samples, and the test results in detail are shown in Table 1.

3.2. Fitting by Using Original Conductivity Functions. In relevant literature reports $[15,18,19,28]$, to obtain the parameters in the conductivity function, the original conductivity function is used to fit the test data of the conductivity in the software Origin. To thoroughly analyze the difference in the fitting results of the eight functions above, after an exposition about the conductivity functions, the experimental XLPE data are fitted by eight common conductivity functions in the software Origin. The fitting principle is the least square principle for minimizing the sum of the square of the absolute errors. The fitting results by using the original conductivity functions are shown in formulas (13)-(20).

$$
\begin{gathered}
\gamma_{\mathrm{pf}}=4.12 \times 10^{-7} \exp \left(-\frac{6173.5596}{T}\right) \exp \left(\frac{0.30718 \sqrt{E}}{T}\right), \\
\gamma_{s}=\frac{1.73886 \times 10^{-5} T^{2}}{E} \exp \left(-\frac{6295.15988}{T}\right) \exp \left(\frac{0.30718 \sqrt{E}}{T}\right),
\end{gathered}
$$


TABle 1: Test data of conductivity of XLPE.

\begin{tabular}{|c|c|c|c|c|c|c|c|c|c|c|}
\hline \multicolumn{11}{|c|}{ Conductivity $(\mathrm{S} / \mathrm{m})$} \\
\hline $\mathrm{T}\left({ }^{\circ} \mathrm{C}\right)$ & $\mathrm{E}(\mathrm{kv} / \mathrm{mm})$ & 10 & 15 & 20 & 25 & 30 & 35 & 40 & 45 & 50 \\
\hline \multirow{5}{*}{22} & 1 & $3.85 \times 10^{-15}$ & $4.15 \times 10^{-15}$ & $4.97 \times 10^{-15}$ & $6.87 \times 10^{-15}$ & $9.34 \times 10^{-15}$ & $1.13 \times 10^{-14}$ & $1.45 \times 10^{-14}$ & $2.10 \times 10^{-14}$ & $3.43 \times 10^{-14}$ \\
\hline & 2 & $4.27 \times 10^{-15}$ & $5.68 \times 10^{-15}$ & $7.00 \times 10^{-15}$ & $9.08 \times 10^{-15}$ & $1.13 \times 10^{-14}$ & $1.41 \times 10^{-14}$ & $1.93 \times 10^{-14}$ & $2.63 \times 10^{-14}$ & $4.16 \times 10^{-14}$ \\
\hline & 3 & $4.54 \times 10^{-15}$ & $4.52 \times 10^{-15}$ & $6.17 \times 10^{-15}$ & $7.97 \times 10^{-15}$ & $1.01 \times 10^{-14}$ & $1.29 \times 10^{-14}$ & $1.75 \times 10^{-14}$ & $2.53 \times 10^{-14}$ & $3.97 \times 10^{-14}$ \\
\hline & 4 & $4.63 \times 10^{-15}$ & $4.74 \times 10^{-15}$ & $6.43 \times 10^{-15}$ & $8.39 \times 10^{-15}$ & $1.02 \times 10^{-14}$ & $1.33 \times 10^{-14}$ & $1.97 \times 10^{-14}$ & $2.71 \times 10^{-14}$ & $3.81 \times 10^{-14}$ \\
\hline & Average & $4.32 \times 10^{-15}$ & $4.77 \times 10^{-15}$ & $6.14 \times 10^{-15}$ & $8.08 \times 10^{-15}$ & $1.02 \times 10^{-14}$ & $1.29 \times 10^{-14}$ & $1.77 \times 10^{-14}$ & $2.49 \times 10^{-14}$ & $3.84 \times 10^{-14}$ \\
\hline \multirow{5}{*}{40} & 1 & $9.05 \times 10^{-15}$ & $1.21 \times 10^{-14}$ & $1.47 \times 10^{-14}$ & $1.81 \times 10^{-14}$ & $2.28 \times 10^{-14}$ & $2.86 \times 10^{-14}$ & $3.97 \times 10^{-14}$ & $5.46 \times 10^{-14}$ & $7.83 \times 10^{-14}$ \\
\hline & 2 & $1.25 \times 10^{-14}$ & $1.43 \times 10^{-14}$ & $1.74 \times 10^{-14}$ & $2.22 \times 10^{-14}$ & $2.80 \times 10^{-14}$ & $3.63 \times 10^{-14}$ & $4.86 \times 10^{-14}$ & $6.23 \times 10^{-14}$ & $8.37 \times 10^{-14}$ \\
\hline & 3 & $9.99 \times 10^{-15}$ & $1.26 \times 10^{-14}$ & $1.58 \times 10^{-14}$ & $2.00 \times 10^{-14}$ & $2.49 \times 10^{-14}$ & $3.28 \times 10^{-14}$ & $4.38 \times 10^{-14}$ & $5.81 \times 10^{-14}$ & $8.02 \times 10^{-14}$ \\
\hline & 4 & $1.15 \times 10^{-14}$ & $1.32 \times 10^{-14}$ & $1.61 \times 10^{-14}$ & $2.11 \times 10^{-14}$ & $2.76 \times 10^{-14}$ & $3.61 \times 10^{-14}$ & $4.65 \times 10^{-14}$ & $5.94 \times 10^{-14}$ & $8.22 \times 10^{-14}$ \\
\hline & Average & $1.08 \times 10^{-14}$ & $1.31 \times 10^{-14}$ & $1.60 \times 10^{-14}$ & $2.03 \times 10^{-14}$ & $2.58 \times 10^{-14}$ & $3.34 \times 10^{-14}$ & $4.47 \times 10^{-14}$ & $5.86 \times 10^{-14}$ & $8.11 \times 10^{-14}$ \\
\hline \multirow{5}{*}{55} & 1 & $2.47 \times 10^{-14}$ & $2.81 \times 10^{-14}$ & $3.00 \times 10^{-14}$ & $3.51 \times 10^{-14}$ & $4.66 \times 10^{-14}$ & $6.47 \times 10^{-14}$ & $8.16 \times 10^{-14}$ & $1.04 \times 10^{-13}$ & $1.47 \times 10^{-13}$ \\
\hline & 2 & $2.80 \times 10^{-14}$ & $3.12 \times 10^{-14}$ & $3.80 \times 10^{-14}$ & $4.74 \times 10^{-14}$ & $5.68 \times 10^{-14}$ & $7.16 \times 10^{-14}$ & $8.86 \times 10^{-14}$ & $1.18 \times 10^{-13}$ & $1.71 \times 10^{-13}$ \\
\hline & 3 & $2.60 \times 10^{-14}$ & $2.97 \times 10^{-14}$ & $3.13 \times 10^{-14}$ & $4.00 \times 10^{-14}$ & $5.09 \times 10^{-14}$ & $6.57 \times 10^{-14}$ & $9.12 \times 10^{-14}$ & $1.11 \times 10^{-13}$ & $1.57 \times 10^{-13}$ \\
\hline & 4 & $2.71 \times 10^{-14}$ & $3.04 \times 10^{-14}$ & $3.82 \times 10^{-14}$ & $4.39 \times 10^{-14}$ & $5.51 \times 10^{-14}$ & $7.05 \times 10^{-14}$ & $8.79 \times 10^{-14}$ & $1.16 \times 10^{-13}$ & $1.71 \times 10^{-13}$ \\
\hline & Average & $2.65 \times 10^{-14}$ & $2.98 \times 10^{-14}$ & $3.44 \times 10^{-14}$ & $4.16 \times 10^{-14}$ & $5.24 \times 10^{-14}$ & $6.81 \times 10^{-14}$ & $8.73 \times 10^{-14}$ & $1.12 \times 10^{-13}$ & $1.62 \times 10^{-13}$ \\
\hline \multirow{5}{*}{70} & 1 & $5.31 \times 10^{-14}$ & $5.94 \times 10^{-14}$ & $6.67 \times 10^{-14}$ & $7.56 \times 10^{-14}$ & $9.20 \times 10^{-14}$ & $1.13 \times 10^{-13}$ & $1.35 \times 10^{-13}$ & $1.61 \times 10^{-13}$ & $2.08 \times 10^{-13}$ \\
\hline & 2 & $6.56 \times 10^{-14}$ & $6.97 \times 10^{-14}$ & $7.66 \times 10^{-14}$ & $8.71 \times 10^{-14}$ & $1.04 \times 10^{-13}$ & $1.22 \times 10^{-13}$ & $1.55 \times 10^{-13}$ & $1.96 \times 10^{-13}$ & $2.68 \times 10^{-13}$ \\
\hline & 3 & $5.56 \times 10^{-14}$ & $6.03 \times 10^{-14}$ & $7.14 \times 10^{-14}$ & $8.07 \times 10^{-14}$ & $9.66 \times 10^{-14}$ & $1.17 \times 10^{-13}$ & $1.36 \times 10^{-13}$ & $1.70 \times 10^{-13}$ & $2.32 \times 10^{-13}$ \\
\hline & 4 & $6.45 \times 10^{-14}$ & $6.63 \times 10^{-14}$ & $7.33 \times 10^{-14}$ & $8.46 \times 10^{-14}$ & $9.92 \times 10^{-14}$ & $1.21 \times 10^{-13}$ & $1.44 \times 10^{-13}$ & $1.83 \times 10^{-13}$ & $2.57 \times 10^{-13}$ \\
\hline & Average & $5.97 \times 10^{-14}$ & $6.39 \times 10^{-14}$ & $7.20 \times 10^{-14}$ & $8.20 \times 10^{-14}$ & $9.80 \times 10^{-14}$ & $1.18 \times 10^{-13}$ & $1.43 \times 10^{-13}$ & $1.77 \times 10^{-13}$ & $2.41 \times 10^{-13}$ \\
\hline \multirow{5}{*}{85} & 1 & $1.05 \times 10^{-13}$ & $1.09 \times 10^{-13}$ & $1.22 \times 10^{-13}$ & $1.36 \times 10^{-13}$ & $1.59 \times 10^{-13}$ & $1.82 \times 10^{-13}$ & $2.17 \times 10^{-13}$ & $2.87 \times 10^{-13}$ & $4.09 \times 10^{-13}$ \\
\hline & 2 & $1.40 \times 10^{-13}$ & $1.43 \times 10^{-13}$ & $1.56 \times 10^{-13}$ & $1.80 \times 10^{-13}$ & $2.03 \times 10^{-13}$ & $2.36 \times 10^{-13}$ & $2.79 \times 10^{-13}$ & $3.62 \times 10^{-13}$ & $4.50 \times 10^{-13}$ \\
\hline & 3 & $1.19 \times 10^{-13}$ & $1.26 \times 10^{-13}$ & $1.39 \times 10^{-13}$ & $1.56 \times 10^{-13}$ & $1.72 \times 10^{-13}$ & $1.92 \times 10^{-13}$ & $2.41 \times 10^{-13}$ & $3.10 \times 10^{-13}$ & $4.26 \times 10^{-13}$ \\
\hline & 4 & $1.30 \times 10^{-13}$ & $1.40 \times 10^{-13}$ & $1.51 \times 10^{-13}$ & $1.74 \times 10^{-13}$ & $1.99 \times 10^{-13}$ & $2.34 \times 10^{-13}$ & $2.80 \times 10^{-13}$ & $3.42 \times 10^{-13}$ & $4.67 \times 10^{-13}$ \\
\hline & Average & $1.23 \times 10^{-13}$ & $1.30 \times 10^{-13}$ & $1.42 \times 10^{-13}$ & $1.62 \times 10^{-13}$ & $1.83 \times 10^{-13}$ & $2.11 \times 10^{-13}$ & $2.54 \times 10^{-13}$ & $3.25 \times 10^{-13}$ & $4.38 \times 10^{-13}$ \\
\hline \multirow{5}{*}{95} & 1 & $1.79 \times 10^{-13}$ & $1.91 \times 10^{-13}$ & $2.01 \times 10^{-13}$ & $2.18 \times 10^{-13}$ & $2.61 \times 10^{-13}$ & $3.38 \times 10^{-13}$ & $4.39 \times 10^{-13}$ & $6.11 \times 10^{-13}$ & $8.05 \times 10^{-13}$ \\
\hline & 2 & $2.08 \times 10^{-13}$ & $2.19 \times 10^{-13}$ & $2.45 \times 10^{-13}$ & $2.88 \times 10^{-13}$ & $3.53 \times 10^{-13}$ & $4.39 \times 10^{-13}$ & $5.35 \times 10^{-13}$ & $6.63 \times 10^{-13}$ & $8.66 \times 10^{-13}$ \\
\hline & 3 & $1.91 \times 10^{-13}$ & $2.05 \times 10^{-13}$ & $2.18 \times 10^{-13}$ & $2.44 \times 10^{-13}$ & $2.86 \times 10^{-13}$ & $3.69 \times 10^{-13}$ & $4.72 \times 10^{-13}$ & $6.33 \times 10^{-13}$ & $8.26 \times 10^{-13}$ \\
\hline & 4 & $1.99 \times 10^{-13}$ & $2.11 \times 10^{-13}$ & $2.31 \times 10^{-13}$ & $2.76 \times 10^{-13}$ & $3.19 \times 10^{-13}$ & $3.93 \times 10^{-13}$ & $5.09 \times 10^{-13}$ & $6.57 \times 10^{-13}$ & $8.43 \times 10^{-13}$ \\
\hline & Average & $1.94 \times 10^{-13}$ & $2.07 \times 10^{-13}$ & $2.24 \times 10^{-13}$ & $2.56 \times 10^{-13}$ & $3.05 \times 10^{-13}$ & $3.85 \times 10^{-13}$ & $4.89 \times 10^{-13}$ & $6.41 \times 10^{-13}$ & $8.35 \times 10^{-13}$ \\
\hline
\end{tabular}

$\gamma_{h}=\frac{34.22963}{E} \exp \left(-\frac{6137.96649}{T}\right) \sinh \left(\frac{2.60266 \times 10^{-5} E}{T}\right)$

$\gamma_{1}=\frac{1.68681}{E} \exp \left(\frac{-5035.17879}{T}\right) \sinh \left(7.10603 \times 10^{-8} E\right)$,

$\gamma_{2}=6.85048 \times 10^{-8} \exp \left(\frac{-5037.42031}{T}+4.25479 \times 10^{-8} E\right)$,

$\gamma_{3}=1.68632 \times 10^{-15} \exp \left(0.0402 \cdot t+4.26242 \times 10^{-8} E\right)$,

$\gamma_{4}=1.50675 \times 10^{-17} \exp \left(\frac{-5037.91972}{T}\right) E^{1.37052}$,

$\gamma_{5}=3.61588 \times 10^{-25} \exp (0.04019 \cdot t) E^{1.37209}$.

From formulas (13)-(20), it can be noted that the temperature functions of $\gamma_{1}, \gamma_{2}$ and $\gamma_{4}$, and $\gamma_{3}$ and $\gamma_{5}$ are nearly the same. In addition, the electric field functions of $\gamma_{\mathrm{pf}}$ and $\gamma_{s}, \gamma_{2}$ and $\gamma_{3}$, and $\gamma_{4}$ and $\gamma_{5}$ are almost the same. The fitting results are in good agreement with the theoretical analysis of the conductivity functions above. Taking formula (4) as an example, a three-dimensional rendering of the fitting is shown in Figure 1.

In Figure 1, the black dots represent the measured conductivity values for different temperatures and electric fields, and the red surfaces represent the fitted results. Figure 1 indicates that the conductivity varies nonlinearly with the temperature and electric field and changes about two or three orders of magnitude within the range of the electric field and temperature. Therefore, the test data points with a large conductivity playing a key role in minimizing the sum of the square of the absolute errors are very close to the fitting values, resulting in a large relative error for the low conductivity data points and a small relative error for the high conductivity data points.

3.3. Fitting by Using Conductivity Functions after Taking Their Logarithms. To reduce the relative errors of all the tested conductivity data points and fitting points, the variation range of the conductivity is reduced. The method involves taking the logarithm of the conductivity, and some equivalent transformations are made to narrow the gap between a higher 


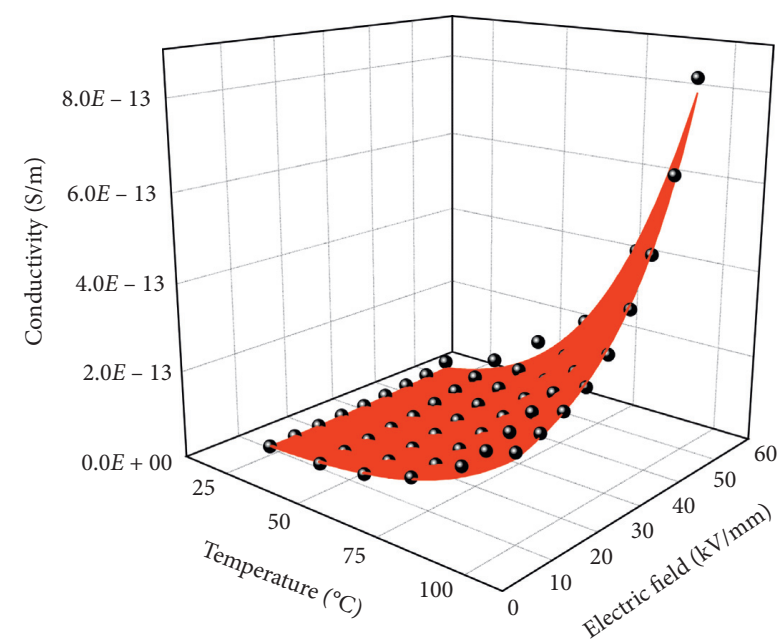

Figure 1: The three-dimensional effect diagram of fitting by formula (4).

conductivity and a lower conductivity. Taking formula (4) as an example, the transformation is shown in the following:

$$
Z=A^{\prime}+C X+\operatorname{In}[\sinh (B Y)]-\operatorname{In} Y,
$$

where $Z=\operatorname{In} \gamma_{1}(E, T), \quad A^{\prime}=\operatorname{In} \gamma_{1,0}, \quad C=\left(\varphi \cdot q / k_{b}\right), \quad X=$ $-(1 / T)$, and $Y=E$.

The logarithms of the other conductivity functions are all taken in the same way as formula (4). The fitting results by using the functions after taking their logarithms are shown in formulas (22)-(29).

$\gamma_{p f}=1.63 \times 10^{-7} \exp \left(-\frac{5645.0821}{T}\right) \exp \left(\frac{0.27686 \sqrt{E}}{T}\right)$,

$\gamma_{s}=\frac{5.38868 \times 10^{-6} T^{2}}{E} \exp \left(-\frac{5694.22846}{T}\right) \exp \left(\frac{0.27686 \sqrt{E}}{T}\right)$,

$\gamma_{h}=\frac{10.33401}{E} \exp \left(-\frac{5673.70388}{T}\right) \sinh \left(\frac{2.52303 \times 10^{-5} E}{T}\right)$,

$\gamma_{1}=\frac{0.95510}{E} \exp \left(-\frac{4879.09861}{T}\right) \sinh \left(7.55817 \times 10^{-8} E\right)$,

$\gamma_{2}=4.62969 \times 10^{-8} \exp \left(\frac{-4879.10373}{T}+4.2107 \times 10^{-8} E\right)$,

$\gamma_{3}=1.28646 \times 10^{-15} \exp \left(0.04423 \cdot t+4.2107 \times 10^{-8} E\right)$,

$\gamma_{4}=4.77325 \times 10^{-15} \exp \left(\frac{-4879.09995}{T}\right) E^{1.01447}$

$$
\gamma_{5}=1.32631 \times 10^{-22} \exp (0.04423 \cdot t) E^{1.01448}
$$

Similar to (13)-(20), formulas (22)-(29) also demonstrate that the temperature functions of $\gamma_{1}, \gamma_{2}$ and $\gamma_{4}$, and $\gamma_{3}$ and $\gamma_{5}$ and the electric field functions of $\gamma_{\mathrm{pf}}$ and $\gamma_{s}, \gamma_{2}$ and $\gamma_{3}$, and $\gamma_{4}$ and $\gamma_{5}$ are almost same. Taking formula (21) as an example, the three-dimensional rendering of the fitting by the conductivity function after taking the logarithm is shown in Figure 2.

The $Z$ axis and $X$ axis in Figure 2 denote the conductivity and temperature transformed according to formula (21). Figure 2 shows that the range of the conductivity after taking the logarithm is approximately from -32 to -28 , which is very small compared to the range of the original conductivity function.

According to the fitting results by the original functions and the functions after taking their logarithms, we find that if the forms of the temperature functions or electric field functions of the formulas are the same, then the coefficients obtained by the fitting are also the same, and only the coefficients $\gamma_{i, 0}$ are different. In addition, fittings by the same formula that use the original function and the function after taking the logarithm always lead to different results.

3.4. Analysis and Discussion. Through the physical meaning of the theoretical conductivity functions, the activation energy $\varphi$, the permittivity $\varepsilon_{r}$, and the jumping distance $d$ are included in the Schottky, Poole-Frenkel, and hopping conductance models. Therefore, the three parameters can be calculated according to the fitting results in formulas (13)-(15) and formulas (22)-(24). Since the temperature functions of empirical formulas (4) and (7) are the same as those of the three theoretical conductivity functions, the activation energy can also be calculated by formulas (4) and (7). For formula (5), the temperature-related term can be viewed as a simplification of temperature-related term in three theoretical functions (1), (2), and (3), and thus the activation energy can also be calculated by formula (5). Therefore, an attempt is made to determine which fitting function is the most accurate by comparing the calculated parameters with the actual values of the material properties. The properties and parameters are shown in Table 2.

From Table 2, it can be seen that the activation energy of XLPE calculated by different fitting formulas is in the range of $0.4-1.1 \mathrm{eV}$, and the discrepancies caused by different formulas are very small. The permittivity calculated by the Schottky and Poole-Frenkel models for the original and logarithm functions is 2.05 and 2.52, respectively, and there is little difference between the real permittivity of XLPE of 2.3. Consequently, it can be concluded that the parameters calculated by the original function and the function after taking the logarithm are approximately the same, and all parameters calculated by different formulas can be accepted.

To further explore the most accurate characterization function of the XLPE conductivity and analyze the fitting effects of different original functions and functions after taking their logarithms, the correlation coefficient R-square and the sum of the squares of the absolute errors and relative 


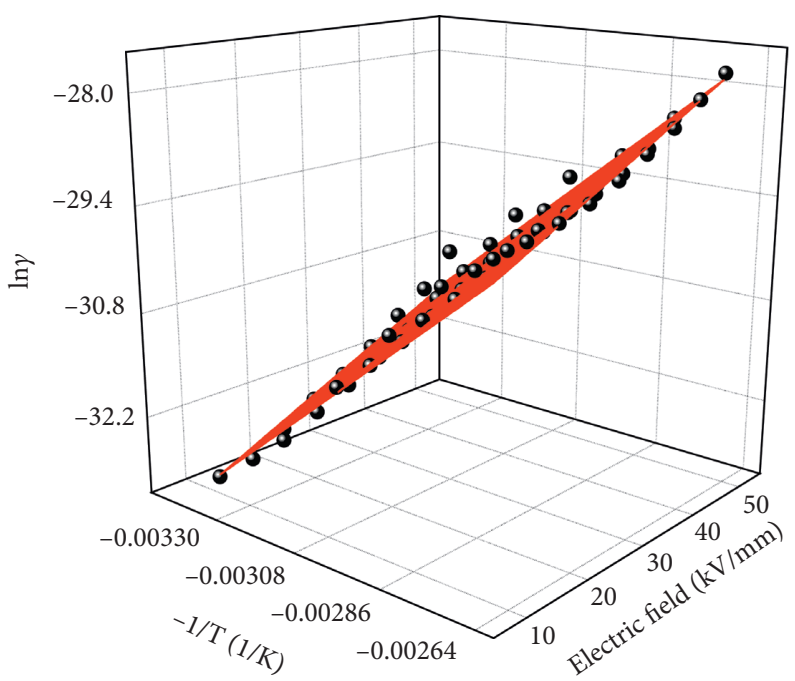

Figure 2: The three-dimensional effect diagram of fitting by formula (21).

TABLE 2: The permittivity, activation energy, and jump distance calculated by different fitting results.

\begin{tabular}{|c|c|c|c|c|c|c|c|c|c|}
\hline Formula & & $(1)$ & $(2)$ & (3) & $(4)$ & (5) & (6) & $(7)$ & $(8)$ \\
\hline Original & $\begin{array}{c}\text { Permittivity } \\
\text { Activation energy }(\mathrm{eV}) \\
\text { Jump distance }(\mathrm{nm})\end{array}$ & $\begin{array}{c}2.05 \\
1.061\end{array}$ & $\begin{array}{c}2.05 \\
0.543\end{array}$ & $\begin{array}{l}0.53 \\
4.49 \\
\end{array}$ & 0.434 & 0.435 & & 0.435 & \\
\hline Logarithm & $\begin{array}{c}\text { Permittivity } \\
\text { Activation energy }(\mathrm{eV}) \\
\text { Jump distance }(\mathrm{nm})\end{array}$ & $\begin{array}{l}2.52 \\
0.97\end{array}$ & $\begin{array}{c}2.52 \\
0.491\end{array}$ & $\begin{array}{l}0.49 \\
4.35\end{array}$ & 0.421 & 0.421 & & 0.421 & \\
\hline
\end{tabular}

errors as judgment standards are extracted. The relevant parameters are shown in Table 3 .

Table 3 indicates that the fitting results obtained by different conductivity functions and fitting methods (original or logarithm) are different. According to the R-square fitting criterion, we find that the best fitting effect achieved by an original conductivity function is formula (4), and the best fitting effect achieved by a conductivity function after taking the logarithm is formula (3); both have been marked in bold. In addition, according to the sum of the square of the absolute errors and relative errors, it can be found that the fitting of the original functions is based on the minimum sum of the square of the absolute errors, and fitting of the functions after taking their logarithms is based on the minimum of the square of the relative errors.

The deviation of the R-square value between formulas (4) and (3) is lower than that between formula (4) and formula (1) or (2), and the deviation of the R-square value between formulas (5) and (3) is larger than that between formula (5) and formula (1) or (2). For formulas (4) to (8) after taking their logarithms, the R-square values decrease from (4) to (8). The fitting results are consistent with the above theoretical analysis and indicate that the accuracy of the empirical formula decreases due to the simplification of the formulas. For the original formulas, the R-square value shows an irregular variation, which is not inconsistent with the theoretical analysis.
Considering that the sum of the relative errors cannot reflect the distribution of the relative error, the relative errors between the testing values and the fitting values by the original functions and the functions after taking their logarithms are described. Taking formula (4) as an example, the results are shown in Figure 3.

It can be seen from Figure 3 that the relative errors of the testing values and fitting values by using the conductivity functions taking their logarithms are evenly distributed in the whole conductivity range. However, the relative errors caused by fitting using the original formulas are smaller when the conductivity is higher and are much larger when the conductivity is lower, which are clearly larger than the relative errors caused by fitting using the formulas after taking their logarithms. The reason for the difference between the relative errors of the conductivity for the original and logarithm functions is that the fitting using an original function is based on the minimum sum of the squares of the absolute errors, and the fitting using a function after taking the logarithm is based on the minimum sum of the squares of the relative errors. In addition, Figure 3 also shows that the fitting after taking the logarithm can take into account all the measured data points of the conductivity, but fitting using the original functions places more attention on data points with higher conductivities, indicating that the fitting method based on the logarithm is more accurate and applicable. 
TABLE 3: Fitting results of XLPE by using eight conductivity functions (original and logarithm).

\begin{tabular}{lccccccccc}
\hline Formula & & $(1)$ & $(2)$ & $(3)$ & $(4)$ & $(5)$ & $(6)$ & $(7)$ & $(8)$ \\
\hline \multirow{4}{*}{ Original } & R-square & 0.9838 & 0.9840 & 0.9930 & $\mathbf{0 . 9 9 4 2}$ & 0.9867 & 0.9885 & 0.9558 & 0.9576 \\
& Sum of squares of absolute errors $\left(\times 10^{-26}\right)$ & 2.27 & 2.25 & 0.978 & $\mathbf{0 . 8 1 1}$ & 1.87 & 1.62 & 6.22 & 5.96 \\
& Sum of squares of relative errors & 2.29 & 2.12 & 1.33 & $\mathbf{0 . 8 3}$ & 1.04 & 2.38 & 3.21 & 3.95 \\
\hline \multirow{2}{*}{ Logarithm } & R-square & 0.9937 & 0.9938 & $\mathbf{0 . 9 9 7 2}$ & 0.9918 & 0.9880 & 0.9852 & 0.9680 & 0.9652 \\
& Sum of squares of absolute errors $\left(\times 10^{-26}\right)$ & 4.43 & 4.27 & $\mathbf{1 . 5 0}$ & 1.92 & 2.05 & 4.28 & 8.36 & 8.79 \\
& Sum of squares of relative errors & 0.51 & 0.50 & $\mathbf{0 . 2 4}$ & 0.70 & 0.91 & 1.24 & 2.40 & 2.80 \\
\hline
\end{tabular}

\section{Electric Field Calculation}

Combining the theoretical analysis with the experimental fitting results of the conductivity functions in Sections 2 and Sections 3 , it is found that the most accurate characterization function is formula (3) taking the logarithm. Besides, the fitting results of different formulas and methods for conductivity may be all acceptable, although there are some differences. However, we do not know the effects of the differences on the simulation of electric field distribution in insulation. In order to study this problem, the steady electric field distribution of HVDC cables under different temperature gradients is simulated by using the software COMSOL Multiphysics.

4.1. Description of the Model. The typical structure of a $320 \mathrm{kV} 500 \mathrm{MW}$ HVDC cable is shown in Figure 4.

In this model, the conductor is copper, the permittivity of the semiconductive shielding layer is 1000, and its conductivity is $0.01 \mathrm{~S} / \mathrm{m}$. The material of the insulation is XLPE in this paper. The relative permittivity of XLPE is 2.3 , and the conductivity is described by the fitting results above.

The temperature of the outside insulation is set to $40^{\circ} \mathrm{C}$ in this paper, and the temperature gradient $\left(0-50^{\circ} \mathrm{C}\right)$ is achieved by changing the temperature of the core conductor. The steady-state temperature of the cable insulation is determined by

$$
T(r)=T_{c}+\frac{\left(\operatorname{In} r-\operatorname{In} R_{c}\right) \cdot\left(T_{\text {out }}-T_{c}\right)}{\operatorname{In} R_{\text {out }}-\operatorname{In} R_{c}},
$$

where $R_{c}$ and $R_{\text {out }}$ are the radii of the inner edge and outer edge of the cable insulation, respectively, $r$ is the radial position in the cable insulation, $T_{c}$ and $T_{\text {out }}$ are the temperatures of the conductor and outside edge of the cable insulation, respectively, and $T(r)$ is the temperature at position $r$.

4.2. Results and Analysis. The amplitude of the voltage is set to $320 \mathrm{kV}$, and the temperature gradients are set to $20^{\circ} \mathrm{C}$, $30^{\circ} \mathrm{C}$, and $50^{\circ} \mathrm{C}$. The steady-state electric field in the insulation of XLPE is calculated, and the results are shown in Figures 5-7, respectively. To make it more conveniently to see the difference of electric field distribution in insulation under different temperature gradients, the scale range of $Y$ axis in Figures 5 to 7 is set up the same.

In addition, it is necessary to apply $1.85 \mathrm{U}_{0} \mathrm{DC}$ test voltage in routine test and type test and $1.45 \mathrm{U}_{0} \mathrm{DC}$ test voltage in prequalification and field installation test.

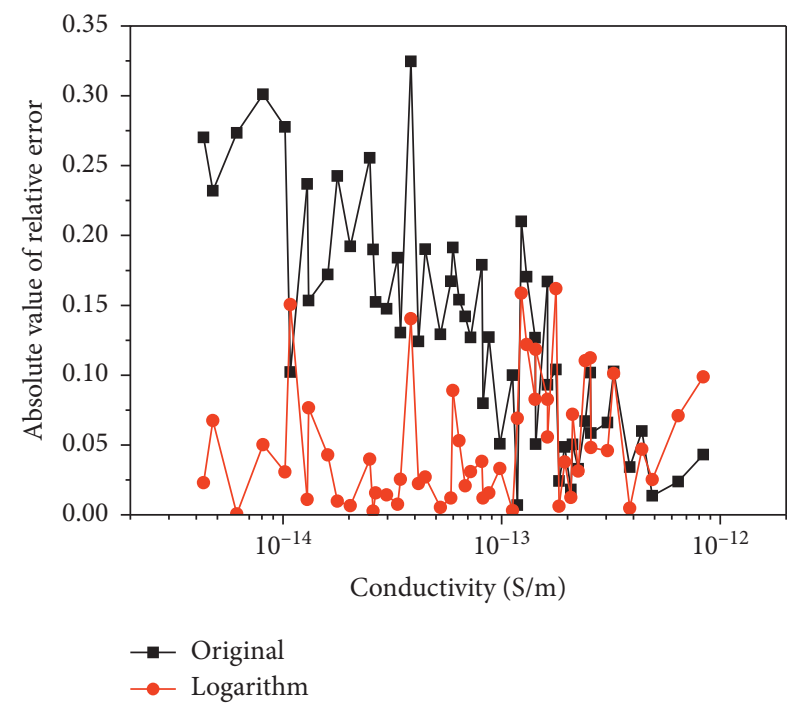

FIGURE 3: Comparison of relative errors of conductivity of XLPE in original and logarithm.

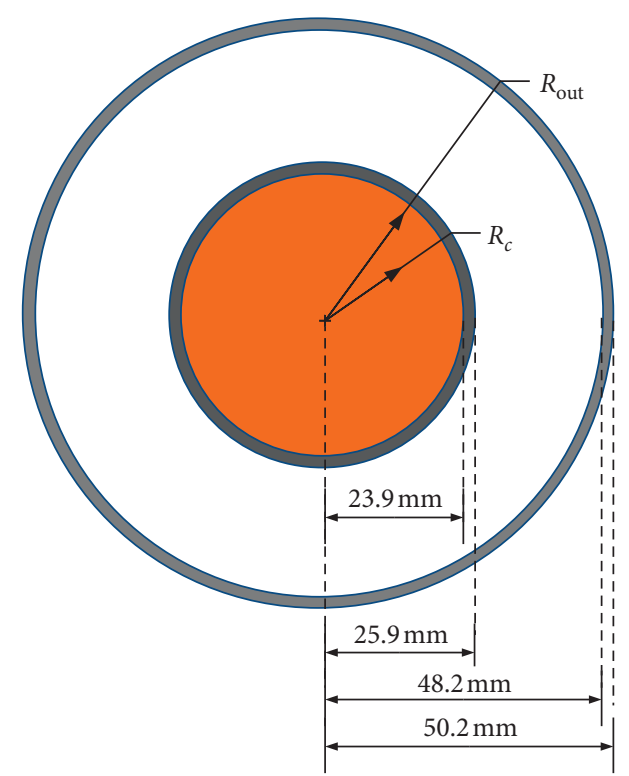

Figure 4: The typical structure of $320 \mathrm{kV}$ HVDC cable.

Therefore, we simulate the electric field under another voltage amplitudes of $1.85 \mathrm{U}_{0}$ which is equal to $592 \mathrm{kV}$, and the temperature gradient is set to $20^{\circ} \mathrm{C}$. The results are shown in Figure 8. 


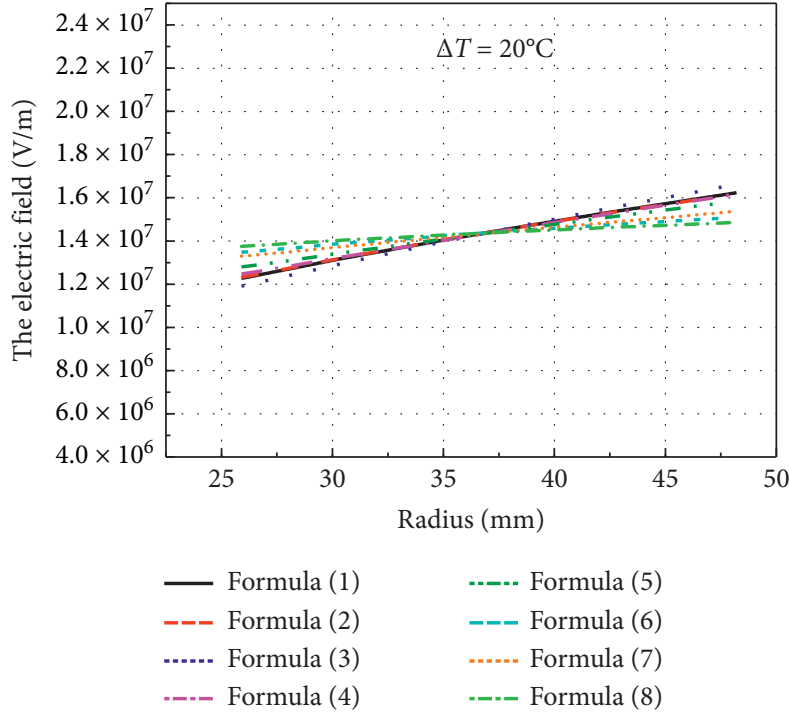

(a)

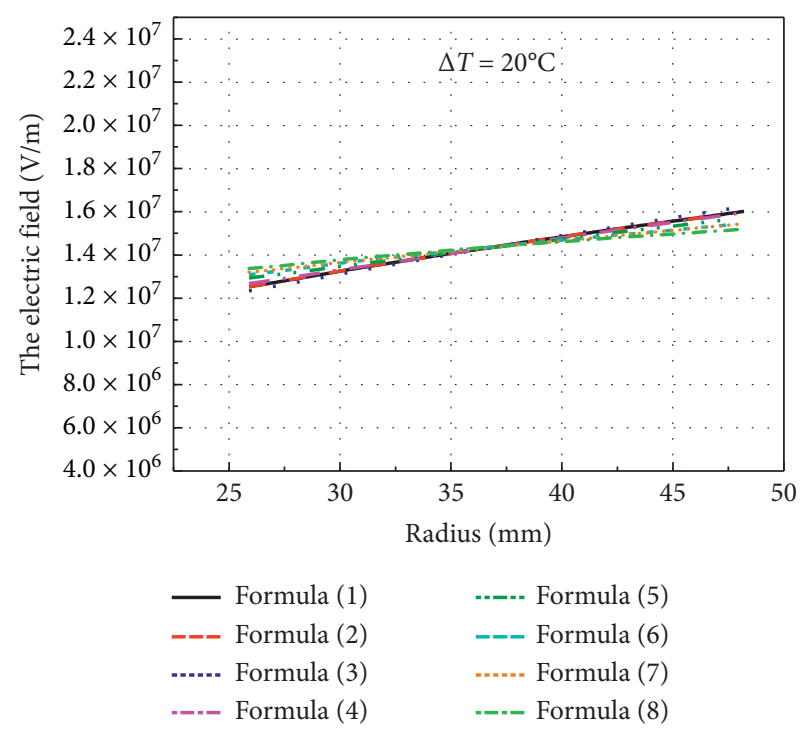

(b)

FIgURE 5: The electric field distribution in the insulation of XLPE by using eight conductivity functions (temperature gradient is $20^{\circ} \mathrm{C}$ ). (a) Simulation by eight original conductivity functions. (b) Simulation by eight conductivity functions taking logarithm.

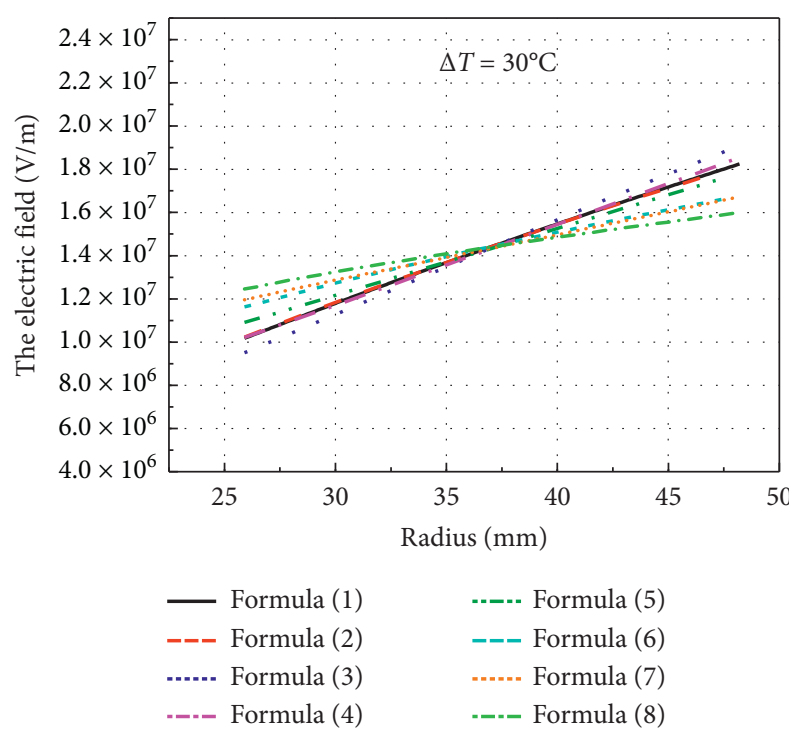

(a)

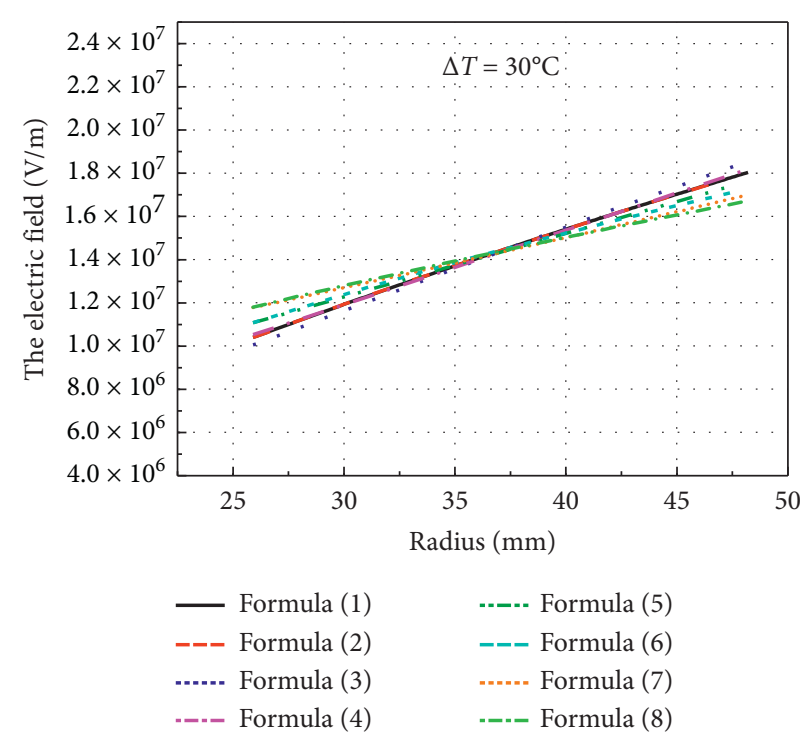

(b)

FIGURE 6: The electric field distribution in the insulation of XLPE by using eight conductivity functions (temperature gradient is $30^{\circ} \mathrm{C}$ ). (a) Simulation by eight original conductivity functions. (b) Simulation by eight conductivity functions taking logarithm.

Figures 5(a)-8(a) describe the steady-state electric field distributions calculated by the original conductivity functions, and Figures $5(\mathrm{~b})-8(\mathrm{~b})$ describe the steady-state electric field calculated by the logarithm of the conductivity function. From Figures 5-8, it can be seen that the electric field distribution is controlled by the conductivity functions. Regardless, whether the temperature gradient is $20^{\circ} \mathrm{C}, 30^{\circ} \mathrm{C}$, or $50^{\circ} \mathrm{C}$ and whether amplitude of the voltage is $320 \mathrm{kV}$ or $592 \mathrm{kV}$, the electric field distribution in the insulation of XLPE is always such that the electric field outside the insulation layer is greater than the electric field inside the insulation layer. In addition, when a simulation is carried out for different conductivity functions, the values of the electric field in the insulation are clearly different.

To quantitatively analyze the difference in the electric field caused by different conductivity functions, some characteristic quantities such as the maximum and minimum of the electric field in the insulation are extracted. In addition, at the same time, taking the electric fields from the original form of formula (4) and the logarithmic form of formula (3), which are the most accurate conductivity functions, as reference values, the relative deviation of the 


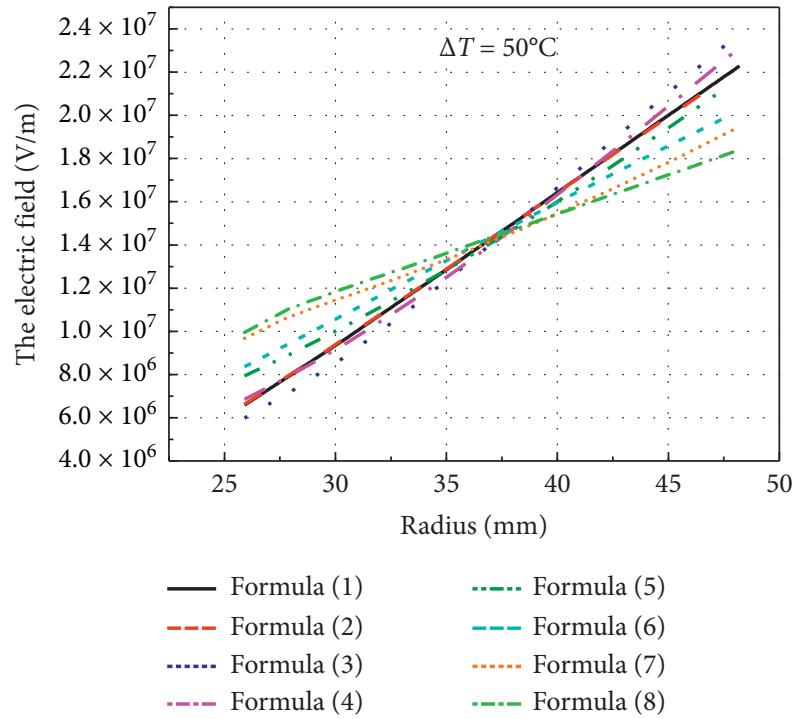

(a)

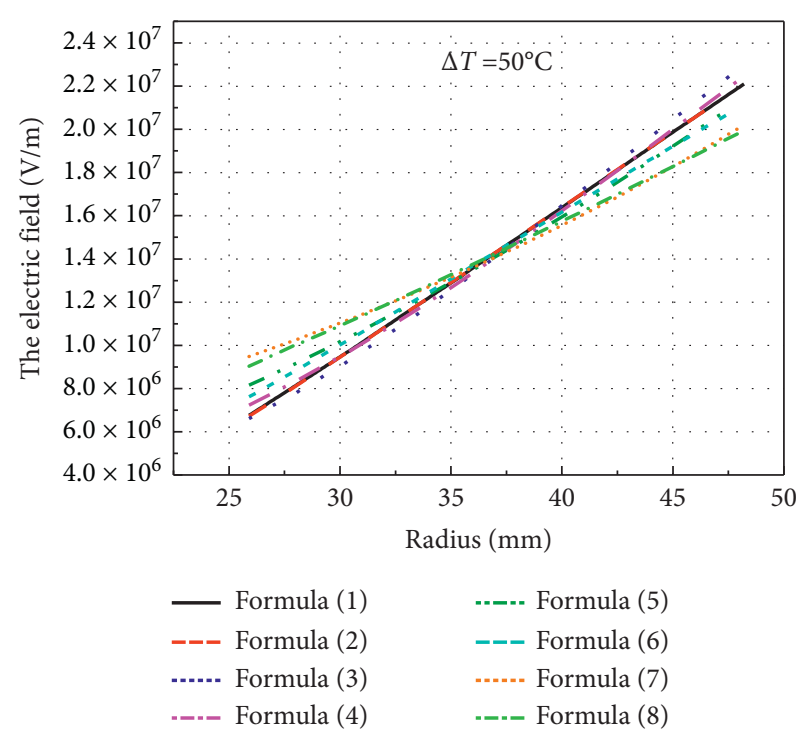

(b)

Figure 7: The electric field distribution in the insulation of XLPE by using eight conductivity functions (temperature gradient is $50^{\circ} \mathrm{C}$ ). (a) Simulation by eight original conductivity functions. (b) Simulation by eight conductivity functions taking logarithm.

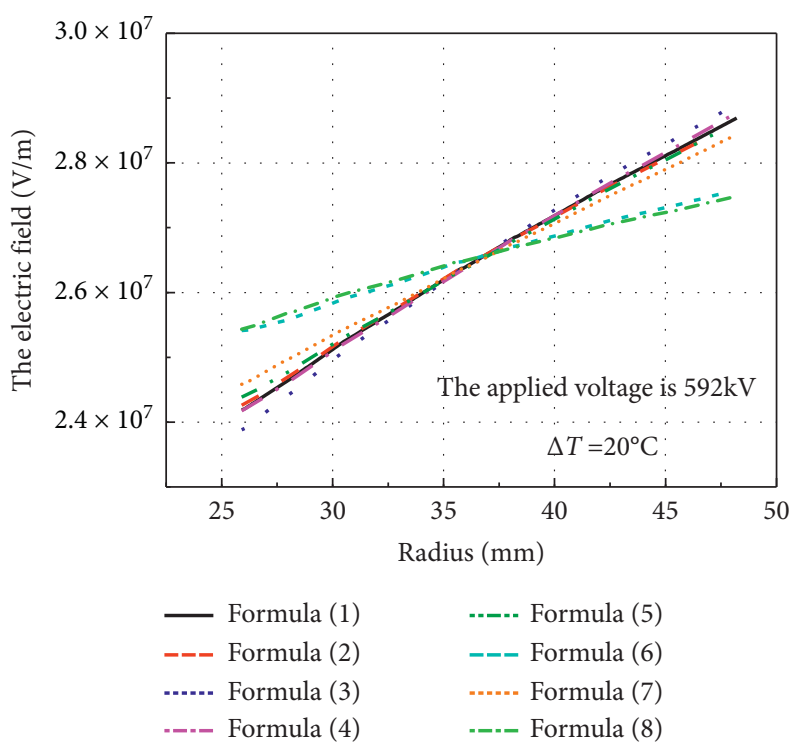

(a)

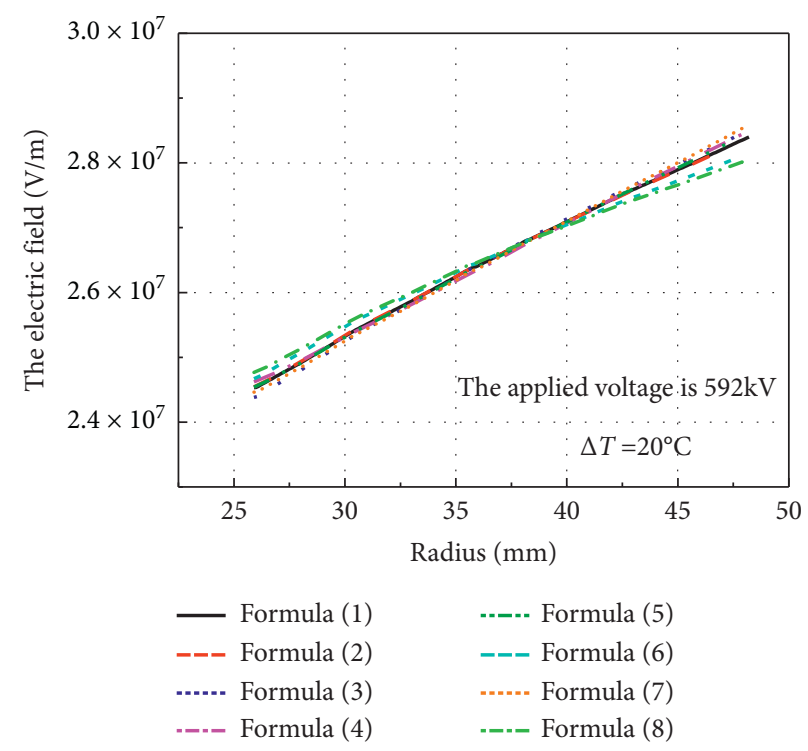

(b)

FIGURE 8: Electric field distribution in the insulation of XLPE by using eight conductivity functions (the voltage amplitude is $592 \mathrm{kV}$ and temperature gradient is $20^{\circ} \mathrm{C}$ ). (a) Simulation of eight original conductivity functions. (b) Simulation of eight conductivity functions after taking their logarithms.

maximum and the relative deviation of the minimum are calculated to assess the difference in the electric field. The results are shown in Tables 4-7.

In addition, the insulation utilization factor, which is used to describe the uniformity of the electric field distribution in insulation [29], defined as formula (31), can also be taken as a characteristic quantity to describe the electric field difference. Thus, the insulation utilization factor and relative deviation from the reference values are also calculated. The results are also shown in Tables 4-7.

$$
\begin{aligned}
\eta & =\frac{E_{a v}}{E_{\max }} \\
& =\frac{\left(U / R_{\text {out }}-R_{c}\right)}{E_{\max }} .
\end{aligned}
$$


TABLE 4: The deviation of the electric field in the insulation of XLPE by using eight conductivity functions (original and logarithm) (temperature gradient is $20^{\circ} \mathrm{C}$ ).

\begin{tabular}{|c|c|c|c|c|c|c|c|c|c|}
\hline Formula & & $(1)$ & $(2)$ & (3) & $(4)$ & $(5)$ & (6) & $(7)$ & $(8)$ \\
\hline \multirow{4}{*}{ Original } & Insulation utilization factor & 0.8841 & 0.8869 & 0.8633 & 0.8875 & 0.9054 & 0.9506 & 0.9334 & 0.9659 \\
\hline & Relative deviation of the insulation utilization factor (\%) & -0.39 & -0.07 & -2.73 & 0.00 & 2.02 & 7.11 & 5.17 & 8.83 \\
\hline & Relative deviation of the maximum (\%) & 0.39 & 0.07 & 2.81 & 0.00 & -1.98 & -6.64 & -4.92 & -8.11 \\
\hline & Relative deviation of the minimum (\%) & -1.57 & -1.20 & -4.47 & 0.00 & 2.62 & 8.11 & 6.66 & 10.30 \\
\hline \multirow{4}{*}{ Logarithm } & Insulation utilization factor & 0.8959 & 0.8966 & 0.8836 & 0.8989 & 0.9125 & 0.9284 & 0.9286 & 0.9444 \\
\hline & Relative deviation of the insulation utilization factor (\%) & 1.40 & 1.47 & 0.00 & 1.73 & 3.27 & 5.07 & 5.08 & 6.88 \\
\hline & Relative deviation of the maximum (\%) & -1.38 & -1.45 & 0.00 & -1.70 & -3.16 & -4.83 & -4.84 & -6.43 \\
\hline & Relative deviation of the minimum (\%) & 1.55 & 1.60 & 0.00 & 2.78 & 4.82 & 6.11 & 7.01 & 8.35 \\
\hline
\end{tabular}

TABLE 5: The deviation of the electric field in the insulation of XLPE by using eight conductivity functions (original and logarithm) (temperature gradient is $30^{\circ} \mathrm{C}$ ).

\begin{tabular}{|c|c|c|c|c|c|c|c|c|c|}
\hline Formula & & $(1)$ & $(2)$ & $(3)$ & $(4)$ & $(5)$ & $(6)$ & $(7)$ & $(8)$ \\
\hline \multirow{4}{*}{ Original } & Insulation utilization factor & 0.7866 & 0.7897 & 0.7515 & 0.7738 & 0.8053 & 0.8564 & 0.8578 & 0.8971 \\
\hline & Relative deviation of the insulation utilization factor (\%) & 1.66 & 2.06 & -2.87 & 0.00 & 4.08 & 10.68 & 10.86 & 15.94 \\
\hline & Relative deviation of the maximum (\%) & -1.64 & -2.02 & 2.96 & 0.00 & -3.92 & -9.65 & -9.80 & -13.75 \\
\hline & Relative deviation of the minimum (\%) & -0.38 & 0.12 & -6.90 & 0.00 & 6.79 & 13.82 & 17.06 & 21.87 \\
\hline \multirow{4}{*}{ Logarithm } & Insulation utilization factor & 0.7955 & 0.7964 & 0.7735 & 0.7891 & 0.8136 & 0.8304 & 0.8438 & 0.8583 \\
\hline & Relative deviation of the insulation utilization factor (\%) & 2.84 & 2.96 & 0.00 & 2.02 & 5.19 & 7.36 & 9.09 & 10.97 \\
\hline & Relative deviation of the maximum (\%) & -2.77 & -2.88 & 0.00 & -1.98 & -4.93 & -6.86 & -8.33 & -9.88 \\
\hline & Relative deviation of the minimum (\%) & 3.47 & 3.44 & 0.00 & 4.69 & 10.25 & 10.25 & 17.22 & 17.38 \\
\hline
\end{tabular}

TABLE 6: The deviation of the electric field in the insulation of XLPE by using eight conductivity functions (original and logarithm) (temperature gradient is $50^{\circ} \mathrm{C}$ ).

\begin{tabular}{|c|c|c|c|c|c|c|c|c|c|}
\hline Formula & & $(1)$ & $(2)$ & $(3)$ & $(4)$ & $(5)$ & $(6)$ & $(7)$ & $(8)$ \\
\hline \multirow{4}{*}{ Original } & Insulation utilization factor & 0.6442 & 0.6471 & 0.6029 & 0.6205 & 0.6607 & 0.7098 & 0.7368 & 0.7798 \\
\hline & Relative deviation of the insulation utilization factor (\%) & 3.81 & 4.28 & -2.83 & 0.00 & 6.47 & 14.38 & 18.74 & 25.67 \\
\hline & Relative deviation of the maximum (\%) & -3.67 & -4.10 & 2.91 & 0.00 & -6.08 & -12.57 & -15.78 & -20.42 \\
\hline & Relative deviation of the minimum (\%) & -3.8 & -3.32 & -12.59 & 0.00 & 15.91 & 22.08 & 41.33 & 45.33 \\
\hline \multirow{4}{*}{ Logarithm } & Insulation utilization factor & 0.6494 & 0.6498 & 0.6247 & 0.6389 & 0.6698 & 0.6788 & 0.7106 & 0.7195 \\
\hline & Relative deviation of the insulation utilization factor (\%) & 3.96 & 4.02 & 0.00 & 2.28 & 7.22 & 8.66 & 13.76 & 15.18 \\
\hline & Relative deviation of the maximum (\%) & -3.81 & -3.87 & 0.00 & -2.23 & -6.74 & -7.97 & -12.09 & -13.18 \\
\hline & Relative deviation of the minimum (\%) & 2.24 & 1.55 & 0.00 & 9.38 & 23.40 & 15.14 & 33.41 & 36.62 \\
\hline
\end{tabular}

TABLE 7: The deviation of the electric field in the insulation of XLPE by using the original and logarithm forms of eight conductivity functions (the voltage amplitude is $592 \mathrm{kV}$ and temperature gradient is $20^{\circ} \mathrm{C}$ ).

\begin{tabular}{|c|c|c|c|c|c|c|c|c|c|}
\hline Formula & & $(1)$ & $(2)$ & $(3)$ & $(4)$ & $(5)$ & $(6)$ & $(7)$ & $(8)$ \\
\hline \multirow{4}{*}{ Original } & Insulation utilization factor & 0.9253 & 0.9275 & 0.9183 & 0.9226 & 0.9272 & 0.9625 & 0.9334 & 0.9659 \\
\hline & Relative deviation of the insulation utilization factor (\%) & 0.29 & 0.53 & -0.47 & 0.00 & 0.50 & 4.32 & 1.17 & 4.69 \\
\hline & Relative deviation of the maximum (\%) & -0.29 & -0.53 & 0.47 & 0.00 & -0.50 & -4.14 & -1.16 & -4.48 \\
\hline & Relative deviation of the minimum (\%) & 0.07 & 0.35 & -1.21 & 0.00 & 0.069 & 5.12 & 1.70 & 5.21 \\
\hline \multirow{4}{*}{ Logarithm } & Insulation utilization factor & 0.9348 & 0.9354 & 0.9313 & 0.9316 & 0.9326 & 0.9433 & 0.9286 & 0.9464 \\
\hline & Relative deviation of the insulation utilization factor (\%) & 0.37 & 0.46 & 0.00 & 0.0302 & 0.14 & 1.29 & -0.29 & 1.6199 \\
\hline & Relative deviation of the maximum (\%) & -0.37 & -0.43 & 0.00 & -0.03 & -0.14 & -1.27 & 0.29 & -1.59 \\
\hline & Relative deviation of the minimum (\%) & 0.62 & 0.67 & 0.00 & 0.98 & 0.66 & 1.19 & 0.32 & 1.57 \\
\hline
\end{tabular}

From formula (31), it can be found that the insulation utilization factor is determined by the average and maximum of the electric field in insulation. The maximum value of the insulation utilization factor is one, and the larger the insulation utilization factor is, the more uniform the electric field distribution in the insulation is.

In Tables 4-7, the reference values marked in italics are determined by the formula with the best fitting effects, and the maximum relative deviations of the characteristic quantities are marked in bold.

From Tables $4-7$, it can be found that the conductivity functions also produce large effects on the results of the insulation utilization factor. The insulation utilization factors are smaller when using the conductivity function with better fitting effect and larger when using the conductivity function with worse fitting effect. Therefore, the most 
accurate conductivity functions should be used to calculate the electric field when designing the structure and materials of HVDC cables. Otherwise, the insulation utilization factor could be larger or smaller than its true value when using inaccurate conductivity functions, which may lead one to misjudge the uniformity of the electric field distribution and then affect the design of the structure and material.

Comparing and analyzing the simulation results in Tables 4-6, it is observed that the deviations of the insulation utilization factor and the maximum and minimum of the electric field increase with the temperature gradient. This result indicates that if the cable temperature gradient is higher, the effects of using different conductivity functions on the electric field of the insulation will also be larger. From the results in Tables 4-7, it could be known that with the increase of the voltage amplitude, the insulation utilization factor increases, and the deviations of the insulation utilization factor and the maximum and minimum of the electric field decrease. The reason is that the higher amplitude of voltage will increase the conductivity nonlinearity of insulating materials, leading to the distribution of the electric field in the insulation more uniform, which is same with the results reported in the literature [30].

In addition, the deviations caused by different logarithmic formulas are smaller than that those caused by the original formulas. And the largest deviations in the electric field in the insulation of XLPE occur for formula (8), whose fitting effects are the worst among the eight formulas. When the temperature gradient is $50^{\circ} \mathrm{C}$, the relative deviations of the insulation utilization factor and maximum and minimum of the electric field calculated by using the original functions are up to $25.67 \%,-20.42 \%$, and $45.33 \%$, respectively. By using the logarithmic functions, the relative deviations of the insulation utilization factor and maximum and minimum of the electric field are up to $15.18 \%,-13.18 \%$, and $36.62 \%$, respectively. When the temperature gradient is $20^{\circ} \mathrm{C}$, the relative deviations of the insulation utilization factor and the maximum and minimum of the electric field calculated by using the original functions are still up to $8.83 \%,-8.11 \%$, and $10.30 \%$, respectively. The deviations of the insulation utilization factor and the maximum and minimum caused by different logarithmic formulas are still up to $6.88 \%,-6.43 \%$, and $8.35 \%$, respectively. The deviations of the electric field caused by different formulas deserve attention when designing HVDC cables or other DC structures. Therefore, it is suggested to use the logarithmic form of formula (3) as the conductivity characterization function when simulating the electric field in the insulation of XLPE cables.

\section{Discussion}

In the present work, it is noteworthy that fitting using the original functions is based on the minimum sum of the squares of the absolute errors, and fitting using the logarithmic functions is based on the minimum sum of the squares of the relative errors. This can be proved by the following derivation. The principle of data fitting is the least square, that is, the minimum sum of the squares of the absolute errors is used to obtain the parameters in conductivity functions. Therefore, the sum of the squares of the absolute error $Q(a, b, c)$ is

$$
\begin{aligned}
Q(a, b, c) & =\sum_{i=1}^{N}\left[f_{i}(a, b, c)-\gamma_{i}\right]^{2} \\
& =\sum_{i=1}^{N}\left(\Delta r_{i}\right)^{2}=\min ,
\end{aligned}
$$

where $N$ is the total number of test points and $f_{i}(a, b, c)$ and $\gamma_{i}$ are the fitting value and testing value of the point $i$, respectively.

The sum of the squares of the relative error is

$$
\begin{aligned}
\operatorname{Qr}(a, b, c) & =\sum_{i=1}^{N}\left[\frac{f_{i}(a, b, c)-\gamma_{i}}{\gamma_{i}}\right] \\
& =\sum_{i=1}^{N}\left(\frac{\Delta r_{i}}{\gamma_{i}}\right)^{2} .
\end{aligned}
$$

When the fitting formulas and the measured value of conductivity use the logarithmic forms, the sum of the squares of the absolute error becomes

$$
\begin{aligned}
Q(a, b, c) & =\sum_{i=1}^{N}\left[\operatorname{In} f_{i}(a, b, c)-\operatorname{In} \gamma_{i}\right]^{2} \\
& =\sum_{i=1}^{N}\left[\operatorname{In} \frac{f_{i}(a, b, c)}{\gamma_{i}}\right]^{2} \\
& =\sum_{i=1}^{N}\left[\operatorname{In}\left(\frac{\Delta r_{i}}{\gamma_{i}}+1\right)\right]^{2} .
\end{aligned}
$$

According to the property of the logarithmic function $y=\ln x, y=0$ when $x=1$. Therefore, when the sum of the squares of the absolute error $\sum_{i=1}^{N}\left[\operatorname{In}\left(\left(\Delta r_{i} / \gamma_{i}\right)+1\right)\right]^{2}$ is minimum, the value of $\operatorname{In}\left(\left(\Delta r_{i} / \gamma_{i}\right)+1\right)$ tends to 0 and $\left(\Delta r_{i} / \gamma_{i}\right)$ tends to 0 . In addition, if the sum of the squares of the relative error $\sum_{i=1}^{N}\left(\Delta r_{i} / \gamma_{i}\right)^{2}$ is minimum, $\left(\Delta r_{i} / \gamma_{i}\right)$ also tends to 0 . Therefore, essentially, the fitting method using logarithmic functions is based on the minimum sum of the squares of the relative error.

As shown in the Table 1, the conductivity of XLPE changes greatly in the entire range of the test electric field and temperature (by about two orders of magnitude). Thus, the data points with larger conductivity play a major role in fitting, resulting in a smaller relative error of the fitting values and tested values. The data points with lower conductivity contribute little to the sum of the squares of the absolute errors, resulting in larger relative errors. In contrast, the conductivity of XLPE changes much less after taking the logarithm; the data points with larger or lower conductivities contribute equally in the fitting, and the relative errors are evenly distributed in the whole conductivity range, as shown in Figure 3. The most important insight from the fitting results is that the logarithmic form of the conductivity is more accurate because the fitting is based on the minimum 
sum of the squares of the relative errors; we should employ the logarithm of the conductivity when obtaining the conductivity functions by fitting in future study.

On the basis of the fitting results and the above analysis, it is important to emphasize that to obtain an accurate conductivity characterization function, the conductivity of the material at different temperatures and field strength should be measured first. Then, the logarithmic forms of some common formulas should be selected to fit the tested results. Finally, according to the correlation coefficient of the fitting and the material parameters calculated from the fitting results, the most accurate formula should be selected. Generally, the theoretical formulas are more accurate than empirical formulas. For the XLPE selected in this paper, the most accurate conductivity function is based on the hopping conductance model because of the good fitting result. For other materials, the conductivities are different, which may cause the most accurate characterization formula to be different.

The simulation results of the electric field distribution of HVDC cables with different conductivity formulas show that when calculating the field distribution in the insulation of HVDC cables or other DC insulation structures, it is necessary to select the most accurate conductivity characterization formula and calculate the electric field with the logarithmic form of the formula. If the characterization formula is inaccurate, the distribution of the electric field in the theoretical calculation may be very different from the actual electric field distribution, especially for an insulation structure with a high temperature gradient. Therefore, it is essential to choose an accurate conductivity characterization formula when calculating the electric field.

\section{Conclusions}

The conductivity of a dielectric is a function of temperature and the electric field, which is usually characterized by the eight functions given in this paper. In the present paper, the source and relationship between eight functions have been expounded. Taking XLPE as an example, the conductivity functions of XLPE are obtained by fitting using the original and logarithmic forms of the eight functions. The electric fields in the insulation are simulated to explore the effects of different fitting functions and methods for the simulation of the electric field. The following conclusions are obtained:

The nonlinear conductivity of insulating materials is characterized by many functions: some are theoretical formulas and have definite physical meanings, while some are empirical formulas, and the theoretical formulas are more accurate than empirical formulas.

The conductivity varies over a range, and fitting using a logarithmic function is more reasonable than using the original function. Fitting using a logarithmic function is based on the minimum squared sum of the relative error, and fitting using an original function is based on the least square of the sum of the absolute errors, which causes a larger relative error for lower conductivity test data points.
There are great differences existing in the electric filed distribution of HVDC cable insulation calculated by different characteristic conductivity functions. The deviations of the electric field simulated by different logarithmic conductivity functions are smaller than those simulated by the original conductivity functions. In addition, with an increase in the temperature gradient of the insulation layer or with an decrease in the amplitude of voltage, the deviations of the electric field caused by different conductivity formulas are also larger.

For the insulation materials of XLPE, the most accurate characterization function that can be used to characterize its nonlinear conductivity is the logarithmic form of formula (3). Therefore, it is suggested to use the logarithmic form of formula (3) as the conductivity characterization function when simulating the electric field in the insulation of XLPE cables.

\section{Data Availability}

The conductivity data used to support the findings of this study are included within the article. For any other information about the article, the corresponding author may be contacted via cysuo@hrbust.edu.cn.

\section{Conflicts of Interest}

The authors declare that they have no conflicts of interest.

\section{Acknowledgments}

This study was supported by the Foundation Research Project of Jiangsu Province (grant no. BK20190187) and the Key Subsidized Projects of the National Natural Science Foundation of China (grant no. 51837003).

\section{References}

[1] H. Ghorbani, M. Jeroense, C.-O. Olsson, and M. Saltzer, "HVDC cable systems-highlighting extruded technology," Institute of Electrical and Electronics Engineers Transactions on Power Delivery, vol. 29, no. 1, pp. 414-421, 2014.

[2] J. Yang, Z. He, H. Pang, and G. Tang, "The hybrid-cascaded DC-DC converters suitable for HVdc applications," Institute of Electrical and Electronics Engineers Transactions on Power Electronics, vol. 30, no. 10, pp. 5358-5363, 2015.

[3] M. S. Khalil, "International research and development trends and problems of HVDC cables with polymeric insulation," Institute of Electrical and Electronics Engineers Electrical Insulation Magazine, vol. 30, no. 6, pp. 35-47, 1997.

[4] T. L. Hanley, R. P. Burford, R. J. Fleming, and K. W. Barber, "A general review of polymeric insulation for use in HVDC cables," Institute of Electrical and Electronics Engineers Electrical Insulation Magazine, vol. 19, no. 1, pp. 13-24, 2003.

[5] M. Marzinotto and G. Mazzanti, "Extruded cables for highvoltage direct current transmission," in Advances in Research and Development, pp. 1-349, IEEE Press Series on Power Engineering, Blackwell Science Publications, Oxford, UK, 2013. 
[6] C. W. Reed, "An assessment of material selection for high voltage DC extruded polymer cables," Institute of Electrical and Electronics Engineers Electrical Insulation Magazine, vol. 33, no. 4, pp. 22-26, 2017.

[7] R. Liu, "Long-distance DC electrical power transmission," Institute of Electrical and Electronics Engineers Electrical Insulation Magazine, vol. 29, no. 5, pp. 37-46, 2013.

[8] Y. Zhou, S. Peng, J. Hu, and J. He, "Polymeric insulation materials for HVDC cables: development, challenges and future perspective," Institute of Electrical and Electronics Engineers Transactions on Dielectrics and Electrical Insulation, vol. 24, no. 3, pp. 1308-1318, 2017.

[9] Y. Ohki, "Development of XLPE-insulated cable for highvoltage dc ubmarine transmission line (1) [News from Japn]," Institute of Electrical and Electronics Engineers Electrical Insulation Magazine, vol. 29, no. 4, pp. 65-67, 2013.

[10] D. Fabiani, G. C. Montanari, C. Laurent et al., "Polymeric HVDC cable design and space charge accumulation. part 1: insulation/semicon interface," Institute of Electrical and Electronics Engineers Electrical Insulation Magazine, vol. 23, no. 6, pp. 11-19, 2007.

[11] S. Campus, D. Fabiani, G. C. Montanari et al., "Feature article - polymeric HVDC cable design and space charge accumulation. Part 2: insulation interfaces," Institute of Electrical and Electronics Engineers Electrical Insulation Magazine, vol. 24, no. 1, pp. 14-24, 2008.

[12] B. R. Varlow, J. Robertson, and K. P. Donnelly, "Nonlinear fillers in electrical insulating materials," IET Science, Measurement \& Technology, vol. 1, no. 2, pp. 96-102, 2007.

[13] R. N. Hampton, "Feature article - some of the considerations for materials operating under high-voltage, direct- current stresses," Institute of Electrical and Electronics Engineers Electrical Insulation Magazine, vol. 24, no. 1, pp. 5-13, 2008.

[14] T. J. Lewis, "The role of electrodes in conduction and breakdown phenomena in solid dielectrics," Institute of Electrical and Electronics Engineers Transactions on Electrical Insulation, vol. EI-19, no. 3, pp. 210-216, 1984.

[15] S. Boggs, D. H. Damon, J. Hjerrild, J. T. Holboll, and M. Henriksen, "Effect of insulation properties on the field grading of solid dielectric dc cable," Institute of Electrical and Electronics Engineers Transactions on Power Delivery, vol. 16, no. 4, pp. 456-461, 2001.

[16] G. Mazzanti and M. Marzinotto, "Relationship between the expressions for electrical resistivity and the field profiles in HVDC cable insulation," in Proceedings of the IEEE International Conference on Electrical Insulation and Dielectrics Phenomenon, pp. 947-950, Fort Worth, TX, USA, October 2016.

[17] Y. Liu, S. Zhang, X. Cao, C. Zhang, and W. Li, "Simulation of electric field distribution in the XLPE insulation of a $320 \mathrm{kV}$ DC cable under steady and time-varying states," Institute of Electrical and Electronics Engineers Transactions on Dielectrics and Electrical Insulation, vol. 25, no. 3, pp. 954-964, 2018.

[18] L. Cao, L. Zhong, J. Gao et al., "Conductivity of HVDC cable insulation materials:case study between xlpe nanocomposite and polymer filled XLPE," in Proceedings of the IEEE International Conference on Electrical Materials and Power Equipment, pp. 349-352, Chongqing, China, April 2019.

[19] C. Suo, Y. Qin, and Z. Li, "Effect of thermal aging on dc conductivity of nano-CB/XLPE insulating composites," in Proceedings of the IEEE International Conference on High Voltage Engineering and Application, Chengdu, China, September 2018.
[20] J. Gu, X. Li, and Y. Yin, "Calculation of electric field and temperature field distribution in MVDC polymeric power cable," in Proceedings of the IEEE International Conference on the Properties and Applications of Dielectric Materials, pp. 105-108, Xi'an, China, July 2009.

[21] J. D. Chen and Z. Y. Liu, Dielectric Physics, pp. 194-230, China Machine Press, Beijing, China, 1982, in Chinese.

[22] O. L. Hestad, F. Mauseth, and R. H. Kyte, "Electrical conductivity of medium voltage XLPE insulated cables," in Proceedings of the IEEE Int. Symp. on Electr. Insul., pp. 376380, San Juan, PR, USA, June 2012.

[23] L. A. Dissado and J. C. Fothcrgill, Electncol Depdation Ond Breakdown in Polymers, pp. 240-260, Peter Peregrinus Ltd for IEE, London, UK, 1992.

[24] C. Reddy and T. Ramu, "On the computation of electric field and temperature distribution in HVDC cable insulation," Institute of Electrical and Electronics Engineers Transactions on Dielectrics and Electrical Insulation, vol. 13, no. 6, pp. 12361244, 2006.

[25] X. Qi, Z. Zheng, and S. Boggs, "Engineering with nonlinear dielectrics," Institute of Electrical and Electronics Engineers Electrical Insulation Magazine, vol. 20, no. 6, pp. 27-34, 2004.

[26] C. C. Reddy and T. S. Ramu, "On the DC conductivity of HVDC cable insulation-cautions in using the empirical models," in Proceedings of the International Symposium on Electrical Insulating Materials, pp. 39-42, Yokkaichi, Japan, June 2008.

[27] B. M. Weedy and D. Chu, "HVDC extruded cables-parameters for determination of stress," Institute of Electrical and Electronics Engineers Transactions on Power Apparatus Systems, vol. PAS-103, no. 3, pp. 662-667, 1984.

[28] S. Hou, M. L. Fu, C. Y. Li, B. Z. Han, C. C. Zhang, and Z. H. Li, "Electric field calculation and analysis of HVDC cable joints with nonlinear materials," in Proceedings of the IEEE International Conference on the Properties and Applications of Dielectric Materials, pp. 184-187, Sydney, Australia, July 2015.

[29] Z. Y. Liu, Power Cable Structure and Design, pp. 139-308, Xi'an Jiaotong University Press, Xi'an, China, 1962, in Chinese.

[30] Z. H. Li, L. L. Liu, H. Zheng, and S. T. Liang, "Simulation on the influence factors of electric field distribution in HVDC cable," in Proceedings of the CSEE, vol. 36, no. 9, pp. 25632571, 2016, in Chinese. 